\title{
NOUVELLe
}

\section{Les nesprines, des protéines de l'enveloppe nucléaire, contrôlent mécaniquement différentes transitions épithélio-mésenchymateuses}

François Sipieter, Louis Laurent, Nicolas Borghi
Université de Paris, CNRS, Institut Jacques Monod, 15 rue Hélène Brion, 75013 Paris, France.

nicolas.borghi@cnrs.fr
> Dans les cellules eucaryotes, le noyau est le réceptacle du matériel génétique. Cet organite, le plus grand de tous, est capable de changer de forme et de position selon les contraintes subies par la cellule, laissant ainsi présager de fonctions plus complexes. Cette adaptation à l'environnement cellulaire est possible grâce au cytosquelette qui assure une connexion entre le noyau et les complexes de protéines d'adhérence cellulaire [1]. Un tel couplage repose en particulier, sur des protéines transmembranaires de l'enveloppe nucléaire, appelées nesprines, qui appartiennent aux complexes LINC (linker of nucleoskeleton and cytoskeleton) qui, comme leur nom l'indique, relient également le cytosquelette au nucléosquelette à l'intérieur du noyau [2] (Figure 1). Les dysfonctions des protéines des complexes LINC perturbent de nombreux processus cellulaires, tels que la migration, la polarisation, la division ou la différenciation dans une variété de contextes. En l'absence des nesprines, ou d'autres protéines des complexes LINC, la transcription de gènes en réponse à des signaux mécaniques extracellulaires est perturbée [3]. Diverses maladies sont ainsi associées à des mutations touchant les gènes codant des protéines de ces complexes [4]. Les mécanismes qui sous-tendent ces maladies restent néanmoins mal compris, car on ignore quels dysfonctionnements cellulaires résultent d'une altération de l'intégrité structurale de ces complexes LINC, de leur capacité à contrôler des signaux biochimiques par la force mécanique (mécano-transduction), ou d'une toute autre fonction non mécanique. Pour tenter de répondre à cette question, nous nous sommes intéressés aux nesprines- 2 géantes (nesprines-2G), qui sont impliquées dans le positionnement du noyau [5].

\section{Le cytosquelette exerce}

sur les nesprines une tension sensible à l'environnement cellulaire

Nous avons développé un biosenseur codé génétiquement qui nous permet de mesurer la tension mécanique subie par les nesprines-2G en microscopie de fluorescence [6] (Figure 1). Dans des cultures de cellules épithéliales ou fibroblastiques, ce biosenseur indique que les nesprines fonctionnelles dans leur interaction avec le cytosquelette, sont sous tension, contrairement aux nesprines porteuses de mutations situées au site d'interaction avec le cytosquelette. À l'aide d'agents pharmacologiques, nous avons également montré que l'altération des réseaux dynamiques d'actine et de microtubules, ainsi que la perte d'adhérence cellulaire, avaient un impact sur la tension des nesprines [7]. Nous avons ainsi montré que le cytosquelette des cellules exerçait une tension constitutive sur les nesprines, lorsque celles-ci adhèrent à leur environnement.

Nous avons ensuite évalué la sensibilité de cette tension à différentes contraintes mécaniques extracellulaires. En faisant migrer des cellules sur un support parsemé d'obstacles séparés par des distances plus courtes que la taille du noyau cellulaire, la compression du noyau provoque un relâchement de la tension du cytosquelette sur les nesprines. En revanche, l'étirement du support sur lequel sont cultivées les cellules provoque proportionnellement une extension du noyau et une augmentation de la tension du cytosquelette sur les nesprines. Enfin, nous avons montré que la tension du cytosquelette sur les nesprines diminue avec la densité cellulaire au sein d'une colonie [7]. La tension sur les nesprines est donc sensible aux contraintes mécaniques subies par les cellules.

\section{La tension des nesprines} varie différemment selon les programmes de transition épithéliomésenchymateuse

Nous avons alors cherché à savoir comment les nesprines répondraient à l'induction de transitions épithélio-mésenchymateuses (TEM). Les TEM sont des processus morphogénétiques omniprésents au cours de l'embryogénèse et de la régénération des tissus. Elles interviennent également dans les cancers épithéliaux : les cellules tumorales perdent leur polarité apico-basale typique, adoptent un comportement migratoire et se dissocient partiellement ou complètement de leurs voisines (Figure 1). 


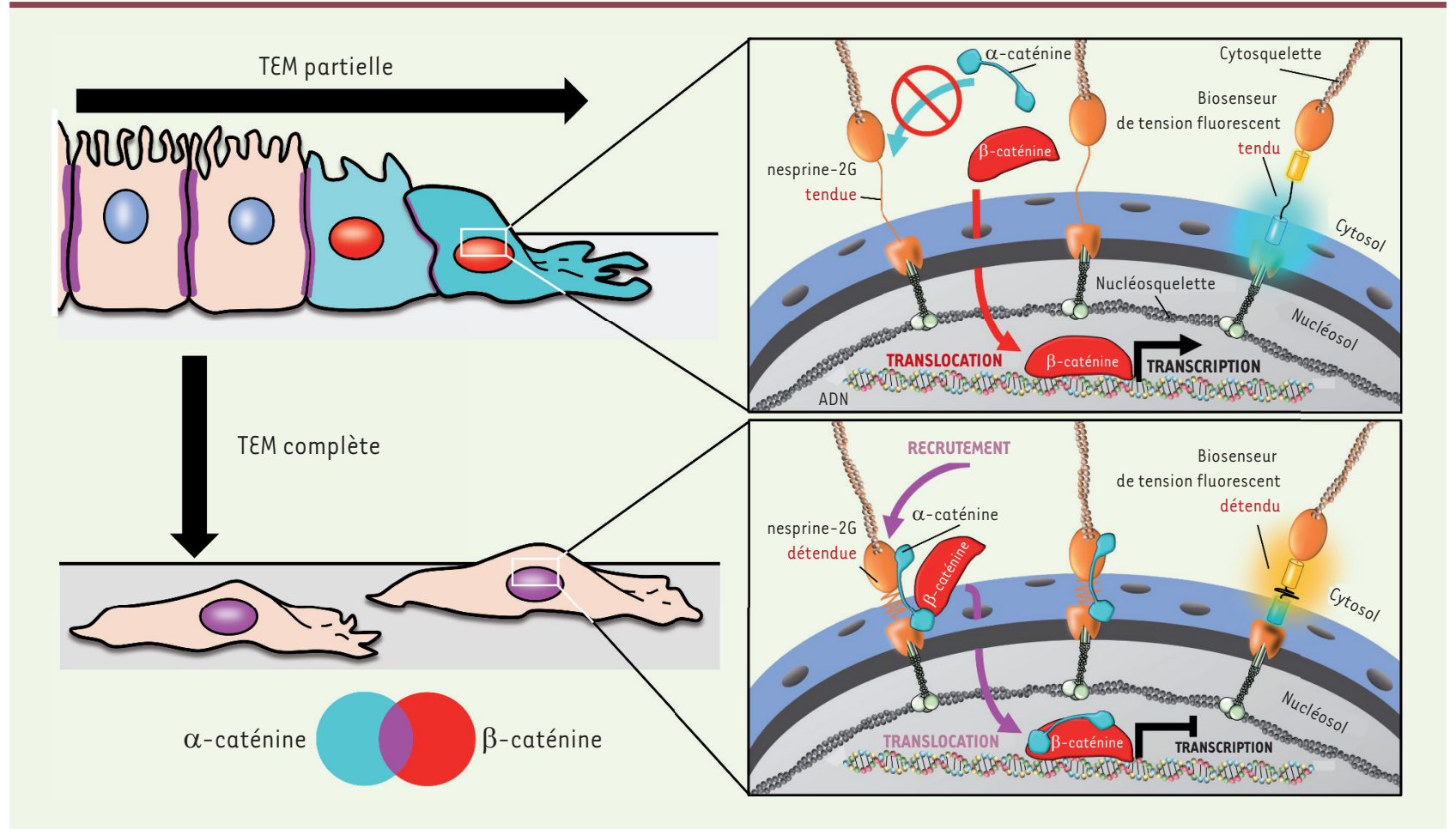

Figure 1. Contrôle de la transcription dépendante de la $\beta$-caténine par la tension des nesprines-2G selon le programme de transition épithéliomésenchymateuse (TEM). Dans des cellules en TEM partielle (en haut), la tension des nesprines-2G empêche le recrutement de l' $\alpha$-caténine à l'enveloppe nucléaire. L' $\alpha$-caténine reste cytoplasmique, et la $\beta$-caténine entre seule dans le noyau, où elle active la transcription de gènes cibles. Dans des cellules en TEM complète (en bas), les nesprines $-2 G$ en conformation détendue permettent le recrutement de l' $\alpha$-caténine à l'enveloppe nucléaire et la translocation des deux caténines dans le noyau, où l' $\alpha$-caténine séquestre la $\beta$-caténine et réduit son activité transcriptionnelle. Dans les deux cas, la tension des nesprines est déterminée expérimentalement avec des biosenseurs codés génétiquement. Grâce à un transfert d'énergie entre fluorophores, la couleur de leur fluorescence dépend de la tension subie par les protéines dans lesquelles ils sont insérés.

En blessant un épithélium en culture, les cellules bordant la blessure adoptent un phénotype mésenchymateux, migratoire, tout en maintenant leurs contacts intercellulaires : il s'agit d'une TEM partielle. Dans ces cellules, nous avons montré que la tension des nesprines augmente, en concordance avec la diminution de la densité cellulaire à cet emplacement. En revanche, la tension des nesprines est insensible à l'exposition des cellules au facteur de croissance HGF (hepatocyte growth factor), qui provoque une TEM complète, caractérisée par la dissociation des cellules épithéliales, puis leur migration individuelle [7]. Ces résultats mettent ainsi en évidence le fait que la tension sur les nesprines varie selon le type de TEM.
La tension des nesprines contrôle la translocation nucléaire de l' $\alpha$ caténine, ce qui influe sur l'activité transcriptionnelle de la $\beta$-caténine Nous nous sommes alors demandé si cette réponse différentielle avait des conséquences fonctionnelles. La $\beta$-caténine est un co-facteur de transcription pour lequel nous avions montré précédemment que sa translocation depuis les complexes d'adhérence intercellulaire, et son activité nucléaire, étaient des évènements précoces des TEM partielle et complète [8]. Nous avons observé que sa partenaire de liaison aux complexes d'adhérence, l' $\alpha$-caténine, est également sujette à une translocation nucléaire lors d'une TEM complète (en exposant les cellules à I'HGF), mais reste dans le cytoplasme lors d'une TEM partielle (en blessant l'épithélium). Grâce à des manipulations génétiques et pharmacologiques, nous avons montré que, lors de la TEM complète, ces deux caténines entraient dans le noyau grâce à une diminution de la tension des nesprines, permettant le recrutement de l' $\alpha$-caténine à l'enveloppe nucléaire, vraisemblablement par le biais d'une interaction directe avec les nesprines elles-mêmes [9]. En revanche, l'augmentation de tension dans les nesprines des cellules en TEM partielle empêche le recrutement de I' $\alpha$-caténine à l'enveloppe nucléaire, et seule la $\beta$-caténine pénètre alors dans le noyau [7] (Figure 1).

Ces résultats posent ainsi la question $\mathrm{du}$ rôle de l' $\alpha$-caténine dans le noyau. Pour 
y répondre, nous avons génétiquement manipulé la translocation nucléaire de l' $\alpha$-caténine et utilisé des biosenseurs de l'activité transcriptionnelle de la $\beta$-caténine codés génétiquement. Nous avons découvert que si l' $\alpha$-caténine nucléaire séquestrait la $\beta$-caténine dans le noyau, elle en réduisait cependant l'activité transcriptionnelle [7].

\section{Conclusion et perspectives}

Nous avons montré que les nesprines sont des mécano-transducteurs: elles sont capables de contrôler des voies de signalisation en réponse à leur état mécanique. En nous fondant sur ces résultats et sur nos travaux précédents [8], nous proposons un modèle selon lequel, en fonction du programme de TEM engagé et de ses répercussions mécaniques sur les nesprines, celles-ci peuvent capturer à l'enveloppe nucléaire les caténines libérées des complexes d'adhérence intercellulaire, et ainsi ajuster subtilement leur translocation nucléaire et leur activité transcriptionnelle. Étant donné l'importance de la voie de signalisation Wnt, dont la $\beta$-caténine est l'effecteur, dans une multitude de processus du développement et dans la tumorigenèse, ces résultats pourraient ouvrir de nouvelles perspectives diagnostiques ou thérapeutiques.

L'implication de la tension des nesprines dans le contrôle de l'accessibilité de la chromatine vient également d'être montrée. Dans des cellules souches épidermiques de souris en culture, une étude propose ainsi que la tension des nesprines, sensible à l'engagement des protéines d'adhérence à la matrice extracellulaire, est importante pour réprimer l'expression de gènes de différenciation épidermique et maintenir une population de cellules progénitrices au cœur de la colonie [10]. Une autre étude propose un rôle de la tension du cytosquelette sur les nesprines dans la différenciation pathologique des myofibroblastes de valves aortiques [11]. Les nesprines sont donc des agents de mécanotransduction dans une large gamme de contextes et par des mécanismes variés, que nous commençons seulement à comprendre. $\diamond$

Nesprins mechanically discriminate between distinct epithelium-tomesenchyme transitions

\section{LIENS D'INTÉRÊT}

Les auteurs déclarent n'avoir aucun lien d'intérêt concernant les données publiées dans cet article.

\section{RÉFÉRENCES}

1. Maniotis AJ, Chen CS, Ingber DE. Demonstration of mechanical connections between integrins, cytoskeletal filaments, and nucleoplasm that stabilize nuclear structure. Proc Natl Acad Sci USA $1997 ; 94: 849-54$.

2. Crisp M, Liu Q, Roux K, et al. Coupling of the nucleus and cytoplasm: role of the LINC complex. J Cell Biol 2006 ; $172: 41-53$.

3. Tajik A, Zhang Y, Wei F, et al. Transcription upregulation via force-induced direct stretching of chromatin. Nat Mater 2016 ; 15 : 1287-96.

4. Janin A, Bauer D, Ratti F, et al. Nuclear envelopathies: a complex LINC between nuclear envelope and pathology. Orphanet J Rare Dis $2017 ; 12$ : 147.

5. Luxton GWG, Gomes $\varepsilon R$, Folker $\varepsilon S$, et al. Linear arrays of nuclear envelope proteins harness retrograde actin flow for nuclear movement. Science 2010 ; 329 : 956-9.

6. Gayrard C, Borghi N. FRET-based molecular tension microscopy. Methods 2016 ; $94: 33-42$.

7. Déjardin T, Carollo PS, Sipieter F, et al. Nesprins are mechanotransducers that discriminate epithelialmesenchymal transition programs. J Cell Biol 2020 ; 219: e201908036.

8. Gayrard C, Bernaudin C, Déjardin T, et al. Src- and confinement-dependent FAK activation causes $\varepsilon$-cadherin relaxation and $\beta$-catenin activity. J Cell Biol 2018 ; 217 : 1063-77.

9. Neumann S, Schneider M, Daugherty RL, et al. Nesprin-2 interacts with (alpha)-catenin and regulates Wnt signaling at the nuclear envelope. J Biol Chem $2010 ; 285: 34932-8$.

10. Carley $\varepsilon$, Stewart RK, Zieman A, et al. The LINC complex transmits integrin-dependent tension to the nuclear lamina and represses epidermal differentiation. elife 2021 ; 10 : e58541.

11. Walker C), Crocini C, Ramirez D, et al. Nuclear mechanosensing drives chromatin remodelling in persistently activated fibroblasts. Nat Biomed Eng $2021 ; 1-15$.

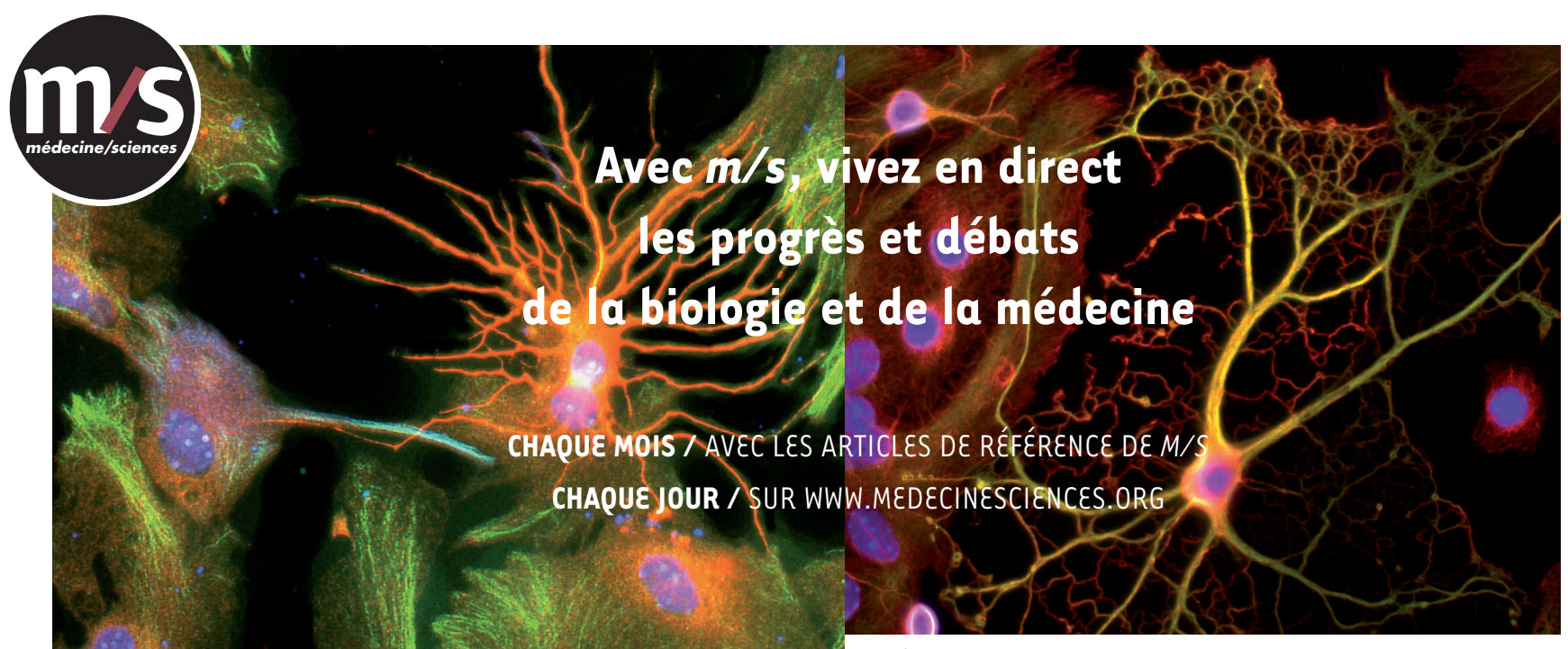

Abonnez-vous sur

WWW.medecinesciences.org 